American Journal of Applied Sciences 9 (3): 405-416, 2012

ISSN 1546-9239

(C) 2012 Science Publications

\title{
Stabilization of a Nonlinear Delay System
}

\author{
${ }^{1,3}$ Walid Arouri, ${ }^{2,3}$ Elyes Maherzi, \\ ${ }^{2,3}$ Mongi Besbes and ${ }^{1,3,4}$ Houda Ben Attia Sethom \\ ${ }^{1}$ National Engineers School of Tunis, Tunisia \\ ${ }^{2}$ Departement of Electrical Engineering, \\ Research Unit on Electrical Machines and Network, Tunisia \\ ${ }^{3}$ Departement of Electrical Engineering, \\ High School of Technology and Compteur Sciences, Tunisia \\ ${ }^{4}$ Departement of Electrical Engineering, \\ Laboratory of Electrical Systems, Tunisia
}

\begin{abstract}
Problem statement: The analysis and control of delayed systems are becoming more and more research topics in progress. This is mainly due to the fact that the delay is frequently encountered in technological systems. This can affect their significantly operations. Most control command laws are based on current digital computers and delays are intrinsic to the process or in the control loop caused by the transmission time control sequences, or computing time. The delay may affect one or more states of the considered system. It may also affect the establishment of the command. Several studies have investigated the stability of delay systems under the assumption that the delay is a variable phenomenon; such variation is considered to be bounded or limited to facilitate analysis of the system. In this study we propose a modelling of delayed system by using the multimodels and switched system theory. The analysis of stability is based on the use of second Lyapunov method. The issued stability conditions are expressed as Bilinear Matrix Inequalities impossible to resolve. That's why we propose the same original relaxations to come over this difficulty, an example of induction machine is given to illustrate over approach. Approach: We propose to use the control theory developed for switched systems to synthesis a control laws for the stabilisation of delays system. Results: We stabilize the induction machine around many operating points despite the non linearities. Conclusion: The developed method is less conservative and less pessimistic than the used classical methods.
\end{abstract}

Key words: Delay systems, lyapunov method, switching system, Linear Matrix Inequalities (LMI), Bilinear Matrix Inequalities (BMI)

\section{INTRODUCTION}

Research in the field of systems controlled via computer networks is growing because of the expansion of computer networks and the development of more robust engines. The economic gains of remote control are more interesting and attract many manufactories.

The analysis and synthesis of delay systems are becoming more and more research topics in progress. This is mainly due to the fact that the delay is frequently encountered in technological systems and can affect their operations significantly. Most are based on current digital computers and delays may occur intrinsically to the process or in the control loop caused by the transmission time control sequences, or computing time. The delay may affect one or more states of the considered system. It may also affect the establishment of the output.
Several studies have modeled the linear systems with delays by differential equations covering both the present and the past states of the system, assuming that the derivative of the vector of states can be explained at every time $\mathrm{t}$.

Other studies consider delay systems as nonlinear and no stationary (Fridman and Shaked, 2002) (Cloosterman et al., 2007) with parameters varying with time or depending on the state of the system. The representation of such variation may be continuous or piecewise continuous (Ariba and Gouaisbaut, 2007).

Approaches have emerged for the analysis of delay systems based on the nature of the delay itself. We can mention: (Zhu and $\mathrm{Hu}, 2009$ ) which assumes that the delay is constant over time. The advantage of such an approach is the reduction of the order of the models obtained and the relative ease to design controllers the whole time the assumption of constant delay is physically questionable. 
Several studies have investigated the stability of delay systems under the assumption that the delay is a variable phenomenon (Gouaisbaut and Peaucelle, 2009; Benchohra et al., 2007) such a change is seen as bounded or limited to facilitate system analysis (Hetel et al., 2007).

The research is carried out to ensure the stability of delay systems are usually around an equilibrium point of the system using, the method of Lyapunov, (Hu et al., 1998) is a temporal method which allows it studying mathematically complex large system dimensions (Migloire, 2004; Labit et al., 2007).

Many studies have aimed at reducing the wide variety of problems of synthesis or analysis in convex optimization problems involving LMI, (Henrion et al., 1999; Teixeira et al., 2003). The problems of control and observation are expressed as Bilinear Matrix Inequalities (BMI) where the resolution of such constraints can have several local solutions, but there are algorithms for local and global optimizations where BMI may lead to a LMI by changing or elimination of well-defined variables (Apkarian and Tuan, 2000).

In Part I of this article, we present an approach for the synthesis of a controller for delayed discrete systems. The delay is considered type variable in time and multiple of the sampling period of the system. In Part II, relaxations are introduced to the transformation of BMI in LMI reaccredit worthy by the current numerical solvers (Matlab $\odot$ or Scilab (C). An illustrative example is given in part II and consists of stabilization of asynchronous machine driven by speed variator via computers network. This example assumes that the delay is due the network. The first part is devoted to the modeling of delay systems. The second part presents the study of the stability of this type of system, which is based on the Lyapunov second method and consequently the relaxation techniques of linear matrix inequalities. The third part is devoted to modeling the induction motor and the study of the stability of the model affected by delays in the order (Kechiche et al., 2011).

\section{MATERIALS AND METHODS}

\section{Delay systems:}

Definition: Delay systems are systems characterized by equations with delays that are introduced to model phenomena in which there is a lag between the action on the system and the system's response to this action. The delay may affect several elements. The delay in the state is written in the form given by Eq. 1: $x(t)=a x(t-\tau)+b u(t)$

The delay in the order, written in the form Eq. 2:

$x(t)=a x(t)+b u(t-\tau)$

The delay in the output is written as Eq. 3:

$y(t-\tau)=c x(t)+d u(t)$

Several models have been associated with delay systems and it appears that several types of systems depending on the nature of the delay.

Stability of delay systems: The stability of linear systems with time-invariant delays is usually studied using the Lyapunov Krasovskii method; this method is very conservative because it generates a lotof LMI conditions difficult to satisfy all. Another approach similar to the approach of Lyapunov Krasovskii has distinguished itself in recent years this is the approach of switched systems (Gouaisbaut and Peaucelle, 2006; Gouaisbaut, 2005).

Delay system to a switching system: The switched system can be described as follows Eq. 4:

$\mathrm{x}(\mathrm{k}+1)=\sum_{\mathrm{i}=1}^{\mathrm{N}} \mu_{\mathrm{i}}(\mathrm{k}) \mathrm{A}_{\mathrm{i}}(\mathrm{k}) \mathrm{x}(\mathrm{k})+\sum_{\mathrm{i}=1}^{\mathrm{N}} \mu_{\mathrm{i}}(\mathrm{k}) \mathrm{B}_{\mathrm{i}}(\mathrm{k}) \mathrm{u}(\mathrm{k})$

where, the parameters $\mu_{\mathrm{i}}(\mathrm{k})$ replace the switching law such as $\Sigma \mu \mathrm{i}=1$.

The return status is written as Eq. 5:

$\mathrm{u}(\mathrm{k})=\sum_{\mathrm{i}=1}^{\mathrm{N}} \mu_{\mathrm{i}}(\mathrm{k}) \mathrm{K}_{\mathrm{i}}(\mathrm{k}) \mathrm{x}(\mathrm{k})$

The closed loop system is given by the following Eq. 6:

$\mathrm{x}(\mathrm{k}+1)=\sum_{\mathrm{i}=1}^{\mathrm{N}} \mu_{\mathrm{i}}(\mathrm{k})\left(\mathrm{A}_{\mathrm{i}}+\mathrm{B}_{\mathrm{i}} \mathrm{k}_{\mathrm{i}}\right) \mathrm{x}(\mathrm{k})$

The formulation of the system with the presence of delays in the control $\mathrm{u}(\mathrm{k})=\mathrm{K}_{\mathrm{i}} \mathrm{x}(\mathrm{k}-\tau)$ is described by switched systems approach that requires an augmented state vector Eq. 7:

$\mathrm{z}(\mathrm{k}))=\left[\mathrm{x}(\mathrm{k}) \ldots . \mathrm{x}\left(\mathrm{k}-\tau_{1}\right)\right]^{\mathrm{T}}$

The formulation of (4) as a switching system is as follows: 
$\mathrm{z}(\mathrm{k}+1)=\overline{\mathrm{A}}_{\varphi}(\mathrm{k}) \mathrm{Z}(\mathrm{k})$

where, the matrix $\overline{\mathrm{A}}_{\varphi}(\mathrm{k})=\overline{\mathrm{A}}_{\mathrm{i}}$ for $\mathrm{i} \in \mathrm{I}=\left\{1, \ldots . . \tau_{1}\right\}$ and:

$$
\overline{\mathrm{A}}_{\mathrm{I}}=\left[\begin{array}{ccccccc}
\mathrm{A}_{\mathrm{d}} & 0 & 0 & -\mathrm{B}_{\mathrm{d}} \mathrm{K} & 0 & . . & 0 \\
\mathrm{I} & 0 & . & . & . & . & 0 \\
0 & \mathrm{I} & 0 & . & . & . & 0 \\
. & 0 & . . & . & . & . & . \\
0 & . & . & . & . & \mathrm{I} & 0
\end{array}\right]
$$

$A_{d}$ block is on the $(i+1)^{\text {éme }}$ th column of $A_{i}$.

After this transformation, system stability is proved using Lyapunov functions, poly quadratic Eq. 8 (Daafouz and Bernussou, 2001):

$\mathrm{v}(\mathrm{k})=\mathrm{z}^{\mathrm{T}} \overline{\mathrm{P}}_{\varphi}(\mathrm{k}) \mathrm{z}(\mathrm{k})$

where, the matrixes $\overline{\mathrm{P}}_{1}, \overline{\mathrm{P}}_{2} \ldots \overline{\mathrm{P}}_{\tau 1}$ are positive definite matrices. The system is asymptotically stable if the matrixes $\quad \overline{\mathrm{P}}_{\mathrm{i}}, \forall \mathrm{i}=1 \ldots \ldots \tau_{1}$ check the satisfies following linear matrix inequality Eq. 9:

$$
\left[\begin{array}{cc}
\overline{\mathrm{P}}_{\mathrm{i}} & \overline{\mathrm{P}}_{\mathrm{i}}^{\mathrm{T}} \overline{\mathrm{P}}_{\mathrm{j}} \\
\overline{\mathrm{P}}_{\mathrm{j}} \overline{\mathrm{P}}_{\mathrm{i}} & \overline{\mathrm{P}}_{\mathrm{j}}
\end{array}\right]>0, \forall(\mathrm{i}, \mathrm{j}) \in \mathrm{I} * \mathrm{I}
$$

The previous system (8) is stable if there exists a state feedback gain $\mathrm{K}$ such that the closed loop system for any bounded delay is stable. We use the equivalent condition to the condition of Lyapunov Eq. 10:

$$
\left[\begin{array}{cc}
G_{i}+G_{i}^{t}-S & G_{i}^{t} A_{i}^{t} \\
A_{i} G_{i} & S
\end{array}\right]>0
$$

For a delay $\tau=\mathrm{i}$, let us apply the method of switched system, the state equation becomes Eq. 11:

$\mathrm{x}(\mathrm{k}+1)=\mathrm{Ax}(\mathrm{k})+\mathrm{BKx}(\mathrm{k}-\mathrm{i})$

Equation 12 can be rewritten as follows:

$$
\left[\begin{array}{c}
\mathrm{x}(\mathrm{k}+1) \\
\mathrm{x}(\mathrm{k}) \\
\cdot \\
\cdot \\
\cdot \\
\mathrm{x}(\mathrm{k}-(\mathrm{i}-1)
\end{array}\right]=\left[\begin{array}{ccccc}
\mathrm{A}_{\mathrm{d}} & \cdot & \cdot & \mathrm{B}_{\mathrm{d}} \mathrm{K} & \cdot \\
\mathrm{I} & 0 & \cdot & \cdot & 0 \\
0 & \mathrm{I} & 0 & \cdot & \cdot \\
0 & \cdot & \mathrm{I} & 0 & \cdot \\
\cdot & \cdot & \cdot & \mathrm{I} & \cdot
\end{array}\right]\left[\begin{array}{c}
\mathrm{x}(\mathrm{k}) \\
\mathrm{x}(\mathrm{k}-1) \\
\cdot \\
\cdot \\
\mathrm{x}(\mathrm{k}-\mathrm{i})
\end{array}\right]
$$

The block $\mathrm{B}_{\mathrm{d}} \mathrm{k}$ is in column $\mathrm{i}$, the switching system adequate representation is as follows given by Eq. 13:

$$
\begin{aligned}
\mathrm{z}(\mathrm{k}+1) & =\mathrm{A}_{\mathrm{i}} \mathrm{z}(\mathrm{k}) \\
\text { with } \mathrm{A}_{\mathrm{i}} & =\left[\begin{array}{ccccc}
\mathrm{A}_{\mathrm{d}} & \cdot & \cdot & \mathrm{B}_{\mathrm{d}} \mathrm{K} & . \\
\mathrm{I} & 0 & \cdot & \cdot & 0 \\
0 & \mathrm{I} & 0 & \cdot & \cdot \\
0 & \cdot & \mathrm{I} & 0 & \cdot \\
\cdot & \cdot & \cdot & \mathrm{I} & .
\end{array}\right]
\end{aligned}
$$

The LMI condition for $\tau=\mathrm{i}$ is Eq. 14:

$\left[\begin{array}{cc}\mathrm{G}_{\mathrm{i}}+\mathrm{G}_{\mathrm{i}}^{\mathrm{t}}-\mathrm{S} & \mathrm{G}_{\mathrm{i}}^{\mathrm{t}} \mathrm{A}_{\mathrm{i}}^{\mathrm{t}} \\ \mathrm{A}_{\mathrm{i}} \mathrm{G}_{\mathrm{i}} & \mathrm{S}\end{array}\right]>0$

Whether $\mathrm{G}_{\mathrm{i}}=\left[\begin{array}{ccccc}\mathrm{g}_{1} & \mathrm{~g}_{2} & \cdot & \cdot & \mathrm{g}_{\mathrm{i}+1} \\ \cdot & \cdot & & & \\ \cdot & & \cdot & & \\ \mathrm{g}_{\mathrm{i}+1} & \cdot & & \cdot & \end{array}\right]$ LMI condition

becomes Eq. 15:

$\left[\begin{array}{cc}\mathrm{G}_{\mathrm{i}}+\mathrm{G}_{\mathrm{i}}^{\mathrm{t}}-\mathrm{S} & \mathrm{G}_{\mathrm{i}}^{\mathrm{t}} \mathrm{A}_{\mathrm{i}}^{\mathrm{t}} \\ \mathrm{A}_{\mathrm{i}} \mathrm{G}_{\mathrm{i}} & \mathrm{S}\end{array}\right]>0$

With:

$$
\begin{aligned}
& \mathrm{A}_{\mathrm{i}} \mathrm{G}_{\mathrm{i}}= \\
& {\left[\begin{array}{ccccc}
\mathrm{A}_{\mathrm{d}} \mathrm{g}_{1} & \mathrm{~A}_{\mathrm{d}} \mathrm{g}_{3}-\mathrm{B}_{\mathrm{d}} \mathrm{Kg}_{4} & . & . & \mathrm{A}_{\mathrm{d}} \mathrm{g}_{\mathrm{i}+1}-\mathrm{B}_{\mathrm{d}} \mathrm{Kg}_{\mathrm{i}+1} \\
\mathrm{~g}_{1} & \mathrm{~g}_{2} & \cdot & . & \mathrm{g}_{\mathrm{i}}+1 \\
\cdot & & & & \\
\cdot & & & & \\
\mathrm{g}_{1}+1 & . & . & . & .
\end{array}\right]}
\end{aligned}
$$

The condition (15) is a BMI unsolvable by current solvers it is essential to introduce relaxations to move to LMI conditions easy to solve it Eq. 16 and 17:

$\mathrm{g}_{\mathrm{i}}=0 \forall \mathrm{i} \in[1, \mathrm{i}]$

$\mathrm{K}_{\mathrm{i}}=\mathrm{R}_{\mathrm{i}+1} * \mathrm{~g}_{\mathrm{i}+1} \quad \mathrm{R}_{\mathrm{i}+1}=\mathrm{K}^{*} \mathrm{~g}_{\mathrm{i}+1}$

Illustrative example:

Consider the following discrete system Eq. 18:

$$
\left\{\begin{array}{l}
{\left[\begin{array}{cc}
=0.99964 & -2.998 \mathrm{e}-005 \\
7.495 \mathrm{e}-005 & 0.999
\end{array}\right] \mathrm{x}+} \\
{\left[\begin{array}{c}
0.0002 \\
7.497 \mathrm{e}-0090
\end{array}\right] \mathrm{u}} \\
\mathrm{y}=\left[\begin{array}{ll}
0 & 1
\end{array}\right] \mathrm{x}
\end{array}\right.
$$


The matrices of the system state switched to a delay $\tau=3$ are written as follows Eq. 19:

$$
\begin{aligned}
\mathrm{z}(\mathrm{k}+1) & =\mathrm{A}_{3} \mathrm{z}(\mathrm{k}) \\
\text { with } \mathrm{A}_{3} & =\left[\begin{array}{cccc}
\mathrm{A}_{\mathrm{d}} & 0 & 0 & -\mathrm{B}_{\mathrm{d}} \mathrm{K} \\
\mathrm{I} & 0 & 0 & 0 \\
0 & \mathrm{I} & 0 & 0 \\
0 & 0 & \mathrm{I} & 0
\end{array}\right]
\end{aligned}
$$

Provider adequate Lyapunov condition is Eq. 20:

$$
\left[\begin{array}{cc}
G_{3}+G_{3}^{t}-S & G_{3}^{t} A_{3}^{t} \\
A_{3} G_{3} & S
\end{array}\right]>0
$$

We set $G_{3}=\left[\begin{array}{llll}g_{1} & g_{2} & g_{3} & g_{4} \\ g_{4} & g_{6} & g_{7} & g_{8} \\ g_{9} & g_{10} & g_{11} & g_{12} \\ g_{13} & g_{14} & g_{15} & g_{16}\end{array}\right]$ the BMI condition for $\tau$ is Eq. 21:

$$
\left[\begin{array}{cc}
\mathrm{G}_{3}+\mathrm{G}_{3}^{\mathrm{t}}-\mathrm{S} & (*)^{\mathrm{t}} \\
(*) & \mathrm{S}
\end{array}\right]>0
$$

With:

$$
\begin{aligned}
& (*)= \\
& {\left[\begin{array}{cccc}
\mathrm{A}_{\mathrm{d}} \mathrm{g}_{1}- & \mathrm{A}_{\mathrm{d}} \mathrm{g}_{2}- & \mathrm{A}_{\mathrm{d}} \mathrm{g}_{3}- & \mathrm{A}_{\mathrm{d}} \mathrm{g}_{4}- \\
\mathrm{B}_{\mathrm{d}} \mathrm{Kg}_{13} & \mathrm{~B}_{\mathrm{d}} \mathrm{Kg}_{14} & \mathrm{~B}_{\mathrm{d}} \mathrm{Kg}_{15} & \mathrm{~B}_{\mathrm{d}} \mathrm{Kg}_{16} \\
\mathrm{~g}_{1} & \mathrm{~g}_{2} & \mathrm{~g}_{3} & \mathrm{~g}_{4} \\
\mathrm{~g}_{5} & \mathrm{~g}_{6} & \mathrm{~g}_{7} & \mathrm{~g}_{8} \\
\mathrm{~g}_{9} & \mathrm{~g}_{10} & \mathrm{~g}_{11} & \mathrm{~g}_{12}
\end{array}\right]}
\end{aligned}
$$

Relaxations are Eq. 22:

$\mathrm{R}_{13}=\mathrm{Kg}_{13}, \mathrm{R}_{14}=\mathrm{Kg}_{14}, \mathrm{R}_{15}=\mathrm{Kg}_{15}$,

$\mathrm{R}_{16}=\mathrm{Kg}_{16}$ et $\mathrm{R}_{13}=\mathrm{R}_{14}=\mathrm{R}_{15}=0$

The introduction of these relaxations can transform BMI (22) in LMI. We find that the system is stable for a gain of state feedback Eq. 23:

$\mathrm{k}_{3}=1.0 \mathrm{e}-004 *$

$\left[\begin{array}{ccccc}-0.1453 & 0.0871 & -0.0510 & -0.0512 & -0.0511 \\ 0 & 0 & 0 & 0 & 0 \\ 0 & 0 & 0 & 0 & 0\end{array}\right]$

For a delay $\in[i, j]$ the system is poly-quadratically stable if the following LMI conditions are satisfied Eq. 24:
Condition for $\tau=\mathrm{i}$

$\left[\begin{array}{cc}\mathrm{G}_{\mathrm{i}}+\mathrm{G}_{\mathrm{i}}^{\mathrm{t}}-\mathrm{S}_{\mathrm{i}} & \mathrm{G}_{\mathrm{i}}^{\mathrm{t}} \mathrm{A}_{\mathrm{i}}^{\mathrm{t}} \\ \mathrm{A}_{\mathrm{i}} \mathrm{G}_{\mathrm{i}} & \mathrm{S}_{\mathrm{i}}\end{array}\right]>0$

For a switching from subsystem $\mathrm{i}$ to subsystem $\mathrm{j}$ Eq. 25:

$\left[\begin{array}{cc}\mathrm{G}_{\mathrm{i}}+\mathrm{G}_{\mathrm{i}}^{\mathrm{t}}-\mathrm{S}_{\mathrm{i}} & \mathrm{G}_{\mathrm{i}}^{\mathrm{t}} \mathrm{A}_{\mathrm{i}}^{\mathrm{t}} \\ \mathrm{A}_{\mathrm{i}} \mathrm{G}_{\mathrm{i}} & \mathrm{S}_{\mathrm{j}}\end{array}\right]>0$

Condition for $\tau=$ j Eq. 26:

$\left[\begin{array}{cc}G_{j}+G_{j}^{t}-S_{j} & G_{j}^{t} A_{j}^{t} \\ A_{j} G_{j} & S_{j}\end{array}\right]>0$

For a switching from subsystem $\mathrm{j}$ to subsystem I Eq. 27:

$\left[\begin{array}{cc}\mathrm{G}_{\mathrm{i}}+\mathrm{G}_{\mathrm{i}}^{\mathrm{t}}-\mathrm{S}_{\mathrm{j}} & \mathrm{G}_{\mathrm{i}}^{\mathrm{t}} \mathrm{A}_{\mathrm{i}}^{\mathrm{t}} \\ \mathrm{A}_{\mathrm{i}} \mathrm{G}_{\mathrm{i}} & \mathrm{S}_{\mathrm{i}}\end{array}\right]>0$

If the delay $\tau=\mathrm{i}<=\mathrm{j}$, we have $A_{i}=\left[\begin{array}{ccccc}A_{d} & \cdot & \cdot & -B_{d} K & 0 . \\ I & 0 & \cdot & \cdot & 0 \\ 0 & I & 0 & \cdot & \cdot \\ 0 & \cdot & I & 0 & \cdot \\ \cdot & \cdot & \cdot & I & \cdot\end{array}\right] B_{d} k$ block is in the column i.

We increase the order of the matrix or elements zero columns' until we reach the same order as $A_{j}(j$ $+1)^{*}(\mathrm{j}+1)$ :

$$
A_{j}=\left[\begin{array}{ccccc}
A_{d} & \cdot & \cdot & -B_{d} K & 0 \\
I & 0 & \cdot & \cdot & 0 \\
0 & I & 0 & \cdot & \cdot \\
0 & \cdot & I & 0 & \cdot \\
\cdot & \cdot & \cdot & I & \cdot
\end{array}\right]
$$

$B_{d} \mathrm{k}$ block is in the jth column of a dimension $(j+1$ $* \mathrm{j}+1)$ whether:

$$
\mathrm{G}_{\mathrm{i}}=\left[\begin{array}{ccccc}
\mathrm{g}_{11} & \mathrm{~g}_{12} & \cdot & \cdot & \mathrm{g}_{\mathrm{i}+1} \\
\cdot & \cdot & & & \\
\cdot & & \cdot & & \\
\mathrm{g}_{\mathrm{i}+1} & \cdot & & \cdot &
\end{array}\right]
$$


Am. J. Applied Sci., 9 (3): 405-416, 2012

$$
G_{j}=\left[\begin{array}{ccccc}
g_{21} & g_{22} & \cdot & \cdot & g_{j+1} \\
\cdot & \cdot & & \\
\cdot & & \cdot & \\
g_{j+1} & \cdot & & \cdot &
\end{array}\right]
$$

BMI conditions become Eq. 28-31:

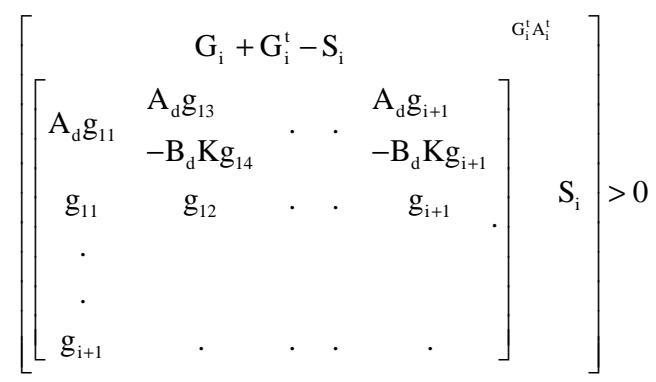

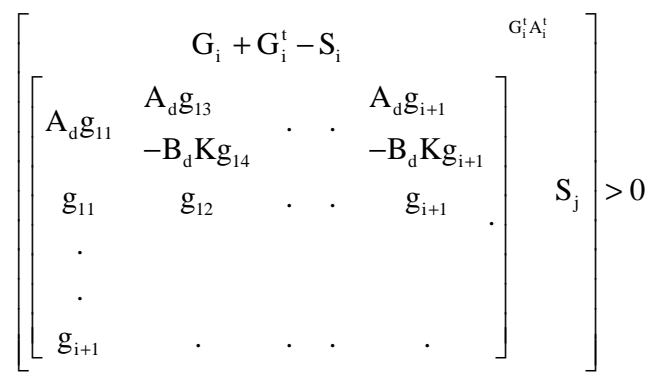

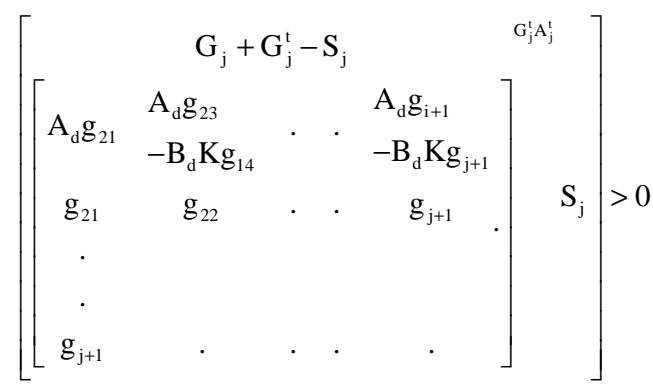

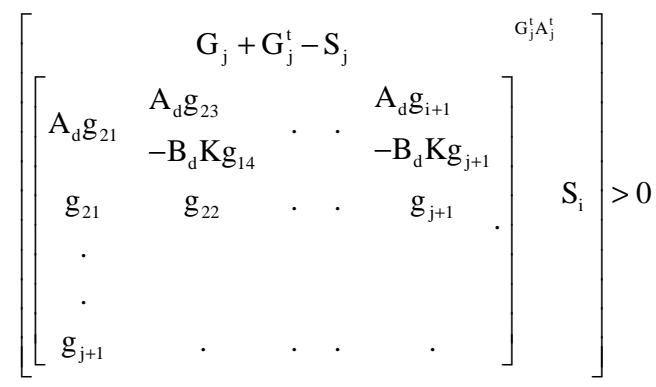

The analysis of these conditions has become difficult as they are of BMI, we introduce the following relaxations Eq. 32-34:
$\mathrm{B}_{\mathrm{d}} \mathrm{K}_{\mathrm{gi}}=0 \forall \mathrm{I} \in[1, \mathrm{i}-1]$

$B_{d} K_{g j}=0 \forall j \in[1, i-1]$

$\mathrm{K}_{\mathrm{i}}=\mathrm{R}_{\mathrm{i}+1} * \mathrm{~g}_{\mathrm{i}+1}{ }^{-1} \mathrm{R}_{\mathrm{i}+1}=\mathrm{K} * \mathrm{~g}_{\mathrm{i}+1}$

Other relaxations can be introduced as $S_{i}=S_{j}$, this optimization is local. The advantage of this hypothesis is the reduction of the number of LMI constraints Eq. 35:

$\left.\left[\begin{array}{ccc}\mathrm{G}_{1}+\mathrm{G}_{1}^{\mathrm{t}}-\mathrm{S}_{1} & \\ \mathrm{~A}_{\mathrm{d}} \mathrm{g}_{11} & \mathrm{~A}_{\mathrm{d}} \mathrm{g}_{21} & \mathrm{~A}_{\mathrm{d}} \mathrm{g}_{31} \\ -\mathrm{B}_{\mathrm{d}} \mathrm{Kg}_{41} & -\mathrm{B}_{\mathrm{d}} \mathrm{Kg}_{51} & -\mathrm{B}_{\mathrm{d}} \mathrm{Kg}_{61} \\ \mathrm{~g}_{11} & \mathrm{~g}_{21} & \mathrm{~g}_{31} \\ \mathrm{~g}_{41} & \mathrm{~g}_{51} & \mathrm{~g}_{61}\end{array}\right] \quad \mathrm{S}_{1}\right]>0$

Illustrative example: Consider the same example above, the delay $\tau$ between switches 1 and 2. BMI conditions are Eq. 36-38:

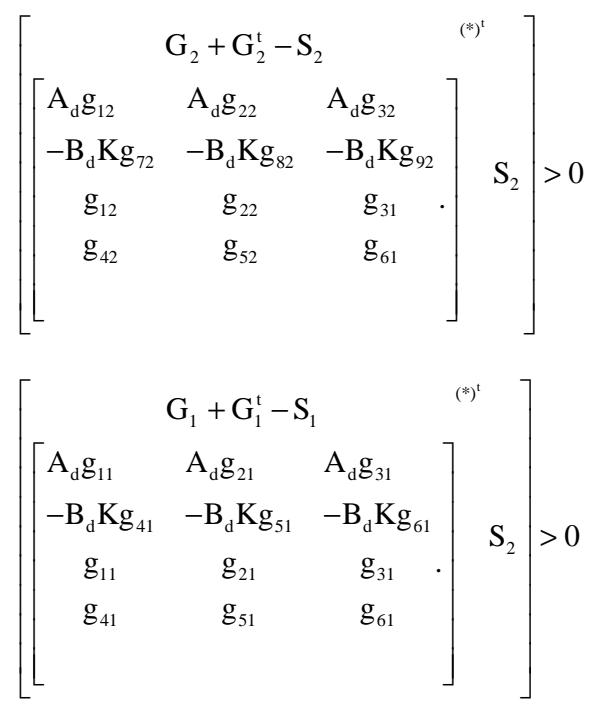

$$
\left.\left[\begin{array}{ccc}
\mathrm{G}_{2}+\mathrm{G}_{2}^{\mathrm{t}}-\mathrm{S}_{2} & \left.{ }^{*}\right)^{\mathrm{t}} \\
\mathrm{A}_{\mathrm{d}} \mathrm{g}_{12}- & \mathrm{A}_{\mathrm{d}} \mathrm{g}_{22}- & \mathrm{A}_{\mathrm{d}} \mathrm{g}_{31}- \\
\mathrm{B}_{\mathrm{d}} \mathrm{Kg}_{72} & \mathrm{~B}_{\mathrm{d}} \mathrm{Kg}_{51} & \mathrm{~B}_{\mathrm{d}} \mathrm{Kg}_{61} \\
\mathrm{~g}_{12} & \mathrm{~g}_{22} & \mathrm{~g}_{32} \\
\mathrm{~g}_{42} & \mathrm{~g}_{52} & \mathrm{~g}_{61}
\end{array}\right] \quad \mathrm{S}_{1}\right]>0
$$

The relaxations are proposed to solve the problem Eq. 39-41:

$\mathrm{R}_{41}=\mathrm{Kg}_{31}, \mathrm{R}_{51}=\mathrm{Kg}_{51}, \mathrm{R}_{61}=\mathrm{Kg}_{61}$ 
$\mathrm{R}_{32}=\mathrm{Kg}_{32}, \mathrm{R}_{72}=\mathrm{Kg}_{72}, \mathrm{R}_{82}=\mathrm{Kg}_{82}$

$\mathrm{R}_{41}=\mathrm{R}_{61}=\mathrm{R}_{72}=\mathrm{R}_{82}=0$

$\mathrm{K}_{1}=\mathrm{R}_{51} \mathrm{~g}_{51}^{-1} \mathrm{~K}_{2}=\mathrm{R}_{92} \mathrm{~g}_{91}^{-1}$

$\mathrm{K}_{1}=\left[\begin{array}{ccccc}16.7204 & 17.5336 & -42.2947 & -41.2243 & -5.9932 \\ 0 & 0 & 0 & 0 & 0 \\ 0 & 0 & 0 & 0 & 0\end{array}\right]$

$\mathrm{K}_{2}=\left[\begin{array}{ccccc}157.8944 & 2.8791 & 20.9317 & 3.3627 & 5.7936 \\ 0 & 0 & 0 & 0 & 0 \\ 0 & 0 & 0 & 0 & 0\end{array}\right]$

Application to the induction machine: We propose to apply this approach to a real industrial system, an induction motor $(5.5 \mathrm{~kW}$ nominal output power, rated voltage $(\mathrm{Y}) 400 \mathrm{~V}$, rated current $(\mathrm{Y}): 11.20 \mathrm{~A}$, rated speed supported: $1445 \mathrm{rpm}$ ) controlled by a variable speed drive via a computer network. Delays prevent the establishment of command sequences:

Induction machine PARK model: The state representation according to the PARK model of the induction machine fed with an inverter is defined by the equation of state as follows: (Castillo-Toledoy et al., 2004; Boucetta, 2008).

In this model:

$\mathrm{x} \in \mathrm{R}^{\mathrm{p}}$ vector state; $\mathrm{u} \in \mathrm{R}^{\mathrm{n}}$ control vector with

$\mathrm{R}_{\mathrm{s}}=$ Stator resistance.

$\mathrm{R}_{\mathrm{r}}=$ Rotor resistance.

$\mathrm{L}_{\mathrm{s}}=$ Stator inductance.

$\mathrm{L}_{\mathrm{r}}=$ rotor inductance.

$\mathrm{M}_{\mathrm{sr}}=$ Mutual inductance.

$\omega_{\mathrm{s}}=$ electrical synchronism pulsation.

$\mathrm{C}_{\mathrm{i}}=$ Vector inverter switching command Eq. 42:

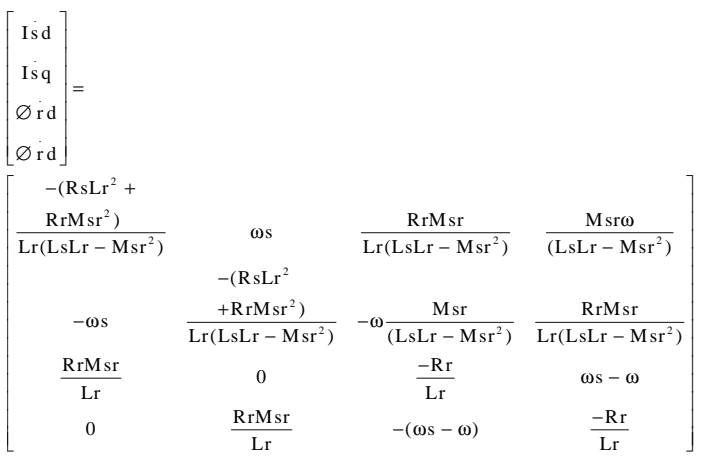

Consequently, referring to previous models we can present a model of the induction machine voltage controlled we impose on the machine to follow a reference speed. The state variables chosen are the stator currents, The rotor flux and the mechanical angular pulsation: $\mathrm{I}_{\mathrm{sd}}, \mathrm{I}_{\mathrm{sq}}, \Phi_{\mathrm{rd}}, \Phi_{\mathrm{rq}}$, the system state is Eq. 43:

$\left[\begin{array}{c}\text { Isd } \\ \text { Isq } \\ \varnothing \dot{r d} \\ \varnothing \dot{r d} \\ \dot{W}\end{array}\right]=$

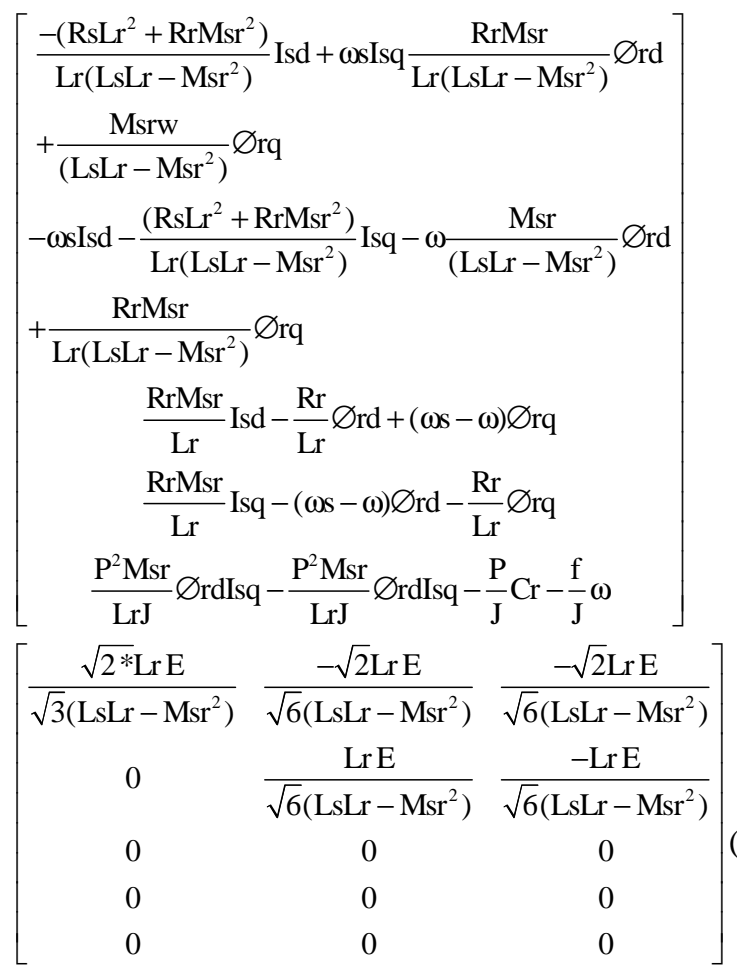

$$
\left[\begin{array}{l}
\mathrm{c}_{1} \\
\mathrm{c}_{2} \\
\mathrm{c}_{3}
\end{array}\right]
$$

The output vector, selected, is Eq 44:

$$
\mathrm{y}(\mathrm{t})=\left[\begin{array}{c}
\varnothing \mathrm{rd} \\
\varnothing \mathrm{rq} \\
\mathrm{W}
\end{array}\right]=\left[\begin{array}{lllll}
0 & 0 & 1 & 0 & 0 \\
0 & 0 & 0 & 1 & 0 \\
0 & 0 & 0 & 0 & 1
\end{array}\right]\left[\begin{array}{c}
\text { Isd } \\
\text { Isq } \\
\varnothing \mathrm{rd} \\
\varnothing \mathrm{rq} \\
\mathrm{W}
\end{array}\right]
$$


The system is nonlinear because there are couplings between the pulsation $\omega$ and the flux $\Phi_{\mathrm{rq}}$, between $\omega$ and the flux $\Phi_{\text {rd }}$, between the current $I_{\text {sq }}$ and $\Phi_{\text {rd }}$ and another between $I_{\text {sd }}$ and flux $\Phi_{\text {rq }}$.

Linearization of the state model of the induction machine: Linearization of the state model of the machine comes from the Jacobian matrix. We determine the operating points and the linear state model around the nominal operating point.The system of linear state machine is Eq. 45:

$$
\left\{\begin{array}{c}
\dot{\tilde{x}}=A \tilde{x}+B \tilde{u} \\
\tilde{y}=C \tilde{x}
\end{array}\right.
$$

With:

$$
\begin{aligned}
& \mathrm{A}= \\
& {\left[\frac{-\left(\mathrm{RsLr}^{2}+\mathrm{RrMsr}^{2}\right)}{\mathrm{Lr}\left(\mathrm{LsLr}-\mathrm{Msr}^{2}\right)}\right.} \\
& \omega \mathrm{s} \\
& -\mathrm{W} \mathrm{s} \\
& \frac{-\left(\mathrm{RsLr}^{2}+\mathrm{RrMsr}^{2}\right)}{\operatorname{Lr}\left(\mathrm{LsLr}-\mathrm{Msr}^{2}\right)} \\
& -\bar{\omega} \frac{\mathrm{Msr}}{\left(\mathrm{LsLr}-\mathrm{Msr}^{2}\right)} \\
& \frac{\mathrm{RrMsr}}{\mathrm{Lr}} \\
& 0 \\
& \frac{-\mathrm{P}^{2} \mathrm{Msr}}{\mathrm{LrJ}} \\
& \mathrm{B}=\left[\begin{array}{c}
\sqrt{2 *} \mathrm{LrE} \\
\sqrt{3}\left(\mathrm{LsLr}-\mathrm{M} \mathrm{sr}^{2}\right) \\
0 \\
0 \\
0 \\
0
\end{array}\right. \\
& \mathrm{C}=\left[\begin{array}{lllll}
0 & 0 & 1 & 0 & 0 \\
0 & 0 & 0 & 1 & 0 \\
0 & 0 & 0 & 0 & 1
\end{array}\right]
\end{aligned}
$$

$\overline{\mathrm{Isq}}, \overline{\mathrm{Isd}}, \overline{\varnothing \mathrm{rq}}, \overline{\varnothing \mathrm{rd}}, \bar{\omega}$ : The nominal operating values of the induction machine.

Control of the induction machine through a data transmission network : The objective is to study the stability of the system despite the occurrence of delays. The sampling period is defined in relation with the smallest constant time which is, $\mathrm{T}_{\mathrm{s}}=\mathrm{L}_{\mathrm{s}} / \mathrm{R}_{\mathrm{s}}$. and the switching period of the inverter given by Eq. 46:

$$
\mathrm{T}_{\mathrm{c}}=1 / \mathrm{f}_{\mathrm{c}}
$$

The system in discrete time is Eq. 47:

$$
\left\{\begin{array}{c}
x(k+1)=A_{d} x(k)+B_{d} u(k) \\
y(k)=C_{d} x(k)+D_{d} u(k)
\end{array}\right.
$$

The delay is attributed to the network so it is introduced into the control $\mathrm{u}(\mathrm{k}-\tau)$. The system (47) becomes Eq. 48-50:

$$
\begin{aligned}
& \left\{\begin{array}{c}
x(k+1)=A_{d} x(k)+B_{d} u(k-\tau) \\
y(k)=C_{d} x(k)+D_{d} u(k-\tau)
\end{array}\right. \\
& \text { or } u(k-\tau)=-K x(k-\tau) \\
& x(k+1)=A_{d} x(k)-B_{d} K x(k-\tau)
\end{aligned}
$$

By applying the method of switching system to find the control matrix K, Such the closed loop system for determining a delay is stable although, we use the equivalent condition to the condition of Lyapunov number (11).

For a delay $\tau=1$ Eq. 51 and 52:

$$
\mathrm{x}(\mathrm{k}+1)=\mathrm{A}_{\mathrm{d}} \mathrm{x}(\mathrm{k})-\mathrm{B}_{\mathrm{d}} \mathrm{Kx}(\mathrm{k}-1)
$$

$\left[\begin{array}{c}\mathrm{x}(\mathrm{k}+1) \\ \mathrm{x}(\mathrm{k})\end{array}\right]=\left[\begin{array}{cc}\mathrm{A}_{\mathrm{d}} & -\mathrm{B}_{\mathrm{d}} \mathrm{K} \\ \mathrm{I} & 0\end{array}\right]\left[\begin{array}{c}\mathrm{x}(\mathrm{k}) \\ \mathrm{x}(\mathrm{k}-1)\end{array}\right]$ 

53:

The matrices of system status switching written Eq.

$$
\mathrm{Z}(\mathrm{k}+1)=\mathrm{A}_{1} \mathrm{Z}(\mathrm{k})
$$

With $A_{1}=\left[\begin{array}{cc}A_{d} & -B_{d} k \\ I & 0\end{array}\right]$

We set $\mathrm{G} 1=\left[\begin{array}{ll}\mathrm{g}_{1} & \mathrm{~g}_{2} \\ \mathrm{~g}_{3} & \mathrm{~g}_{4}\end{array}\right]$ LMI condition for $\tau(\mathrm{k})=1$ is Eq. 54:

$\left[\begin{array}{cc}\mathrm{G}_{1}+\mathrm{G}_{1}^{\mathrm{t}}-\mathrm{S} & \mathrm{G}_{1}^{\mathrm{t}} \mathrm{A}_{1}^{\mathrm{t}} \\ \mathrm{A}_{1} \mathrm{G}_{1} & \mathrm{~S}\end{array}\right]$
$\left[\begin{array}{cc}\mathrm{G}_{1}+\mathrm{G}_{1}^{\mathrm{t}}-\mathrm{S} & (*)^{\mathrm{t}} \\ {\left[\begin{array}{cc}\mathrm{A}_{\mathrm{d}} \mathrm{g}_{1}-\mathrm{B}_{\mathrm{d}} \mathrm{Kg}_{3} & \mathrm{~A}_{\mathrm{d}} \mathrm{g}_{3}-\mathrm{B}_{\mathrm{d}} \mathrm{Kg}_{4} \\ \mathrm{~g}_{1} & \mathrm{~g}_{1}\end{array}\right]>0} & \mathrm{~S}\end{array}\right]>0$

We set $\mathrm{g}_{3}=0$ and $\mathrm{R}_{4}=\mathrm{Kg}_{4}$ LMI condition becomes Eq. 55:

$\left[\begin{array}{cc}\mathrm{G}_{1}+\mathrm{G}_{1}^{\mathrm{t}}-\mathrm{S} & \mathrm{G}_{1}^{\mathrm{t}} \mathrm{A}_{1}^{\mathrm{t}} \\ {\left[\begin{array}{cc}\mathrm{A}_{\mathrm{d}} \mathrm{g}_{1} & \mathrm{~A}_{\mathrm{d}} \mathrm{g}_{3}-\mathrm{B}_{\mathrm{d}} \mathrm{Kg}_{4} \\ \mathrm{~g}_{1} & \mathrm{~g}_{1}\end{array}\right] \quad \mathrm{S}}\end{array}\right]>0$

The system is stable gain state feedback $\mathrm{K}_{1}=\mathrm{R}_{4} \mathrm{~g}_{4}^{-1}$ for a delay $\tau=2$ Eq. 56-57:

$x(k+1)=A_{d} x(k)-B_{d} K x(k-2)$

$\left[\begin{array}{c}\mathrm{x}(\mathrm{k}+1) \\ \mathrm{x}(\mathrm{k}) \\ \mathrm{x}(\mathrm{k}-1)\end{array}\right]=\left[\begin{array}{ccc}\mathrm{A}_{\mathrm{d}} & 0 & -\mathrm{B}_{\mathrm{d}} \mathrm{K} \\ \mathrm{I} & 0 & 0 \\ 0 & \mathrm{I} & 0\end{array}\right]\left[\begin{array}{c}\mathrm{x}(\mathrm{k}) \\ \mathrm{x}(\mathrm{k}-1) \\ \mathrm{x}(\mathrm{k}-2)\end{array}\right]$

The switched system is written Eq. 58:

$\mathrm{Z}(\mathrm{k}+1)=\mathrm{A}_{2} \mathrm{Z}(\mathrm{k})$

With $\quad A_{2}=\left[\begin{array}{ccc}A_{d} & 0 & -B_{d} K \\ \mathrm{I} & 0 & 0 \\ 0 & \mathrm{I} & 0\end{array}\right]$

The Lyapunov condition for $\tau=2$ is Eq. 59:

$\left[\begin{array}{cc}\mathrm{G}_{2}+\mathrm{G}_{2}^{\mathrm{t}}-\mathrm{S} & \mathrm{G}_{2}^{\mathrm{t}}+\mathrm{G}_{2}^{\mathrm{t}} \\ \mathrm{A}_{2}+\mathrm{G}_{2} & \mathrm{~S}\end{array}\right]>0$
We $G_{2}=\left[\begin{array}{lll}g_{12} & g_{22} & g_{32} \\ g_{42} & g_{52} & g_{62} \\ g_{72} & g_{82} & g_{92}\end{array}\right]$ the condition for $\tau=2$ are Eq. 60:

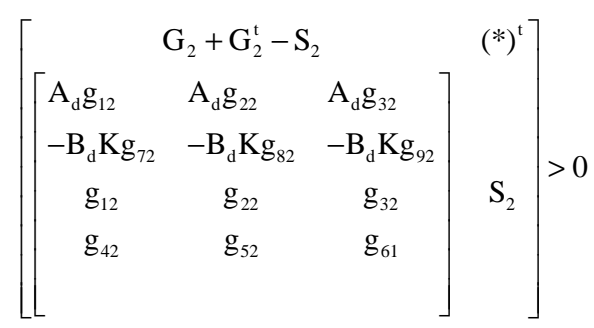

The previous conditions inequalities are BMI, difficult to resolve. We propose the following conditions to come over this difficulty:

$\mathrm{g}_{72}=\mathrm{g}_{82}=0, \mathrm{R}_{92}=\mathrm{Kg}_{92}$

The system is stable for a gain: $\mathrm{K}_{2}=\mathrm{R}_{92} \mathrm{~g}_{92}$. 69:

For a delay $\tau \in[0,2]$, LMI conditions are Eq. 61-

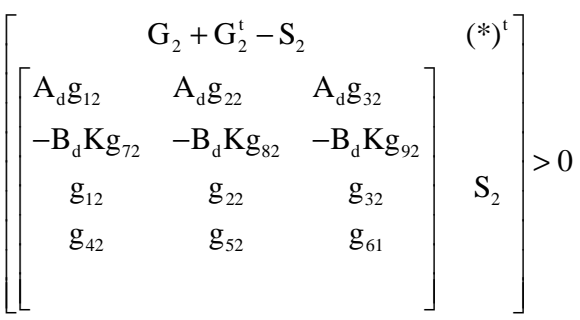

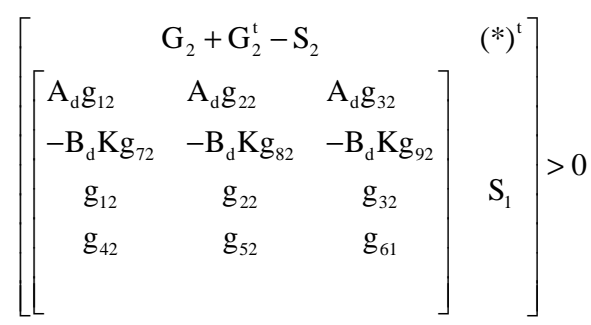

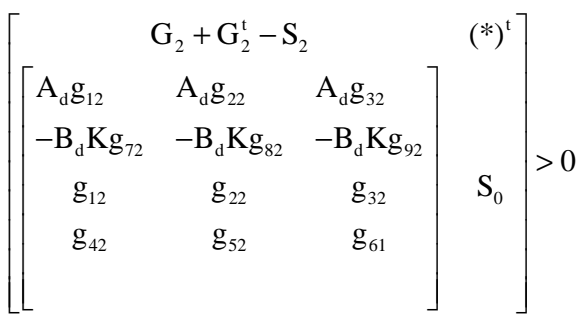


Am. J. Applied Sci., 9 (3): 405-416, 2012

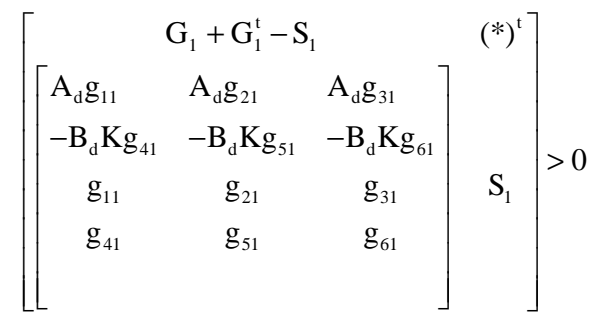

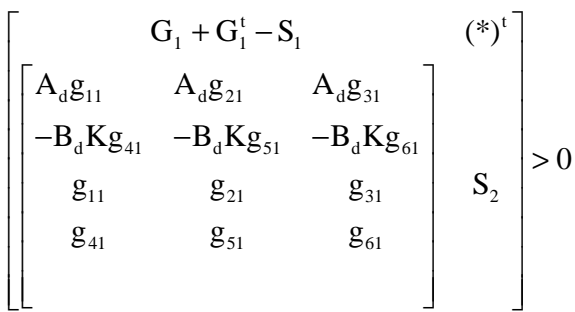

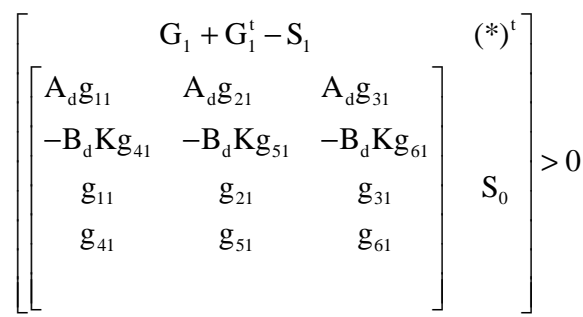

$\left.\left[\begin{array}{ccc}\mathrm{G}_{0}+\mathrm{G}_{0}^{\mathrm{t}}-\mathrm{S}_{0} & \left(^{*}\right)^{\mathrm{t}} \\ \mathrm{A}_{\mathrm{d}} \mathrm{g}_{10} & \mathrm{~A}_{\mathrm{d}} \mathrm{g}_{20} & \mathrm{~A}_{\mathrm{d}} \mathrm{g}_{30} \\ -\mathrm{B}_{\mathrm{d}} \mathrm{Kg}_{10} & -\mathrm{B}_{\mathrm{d}} \mathrm{Kg}_{20} & -\mathrm{B}_{\mathrm{d}} \mathrm{Kg}_{30} \\ \mathrm{~g}_{10} & \mathrm{~g}_{20} & \mathrm{~g}_{30} \\ \mathrm{~g}_{40} & \mathrm{~g}_{50} & \mathrm{~g}_{60}\end{array}\right] \quad \mathrm{S}_{0}\right]>0$

$\left.\left[\begin{array}{cccc}\mathrm{G}_{0}+\mathrm{G}_{0}^{\mathrm{t}}-\mathrm{S}_{0} & & \left(^{*}\right)^{\mathrm{t}} \\ \mathrm{A}_{\mathrm{d}} \mathrm{g}_{10} & \mathrm{~A}_{\mathrm{d}} \mathrm{g}_{20} & \mathrm{~A}_{\mathrm{d}} \mathrm{g}_{30} \\ -\mathrm{B}_{\mathrm{d}} \mathrm{Kg}_{10} & -\mathrm{B}_{\mathrm{d}} \mathrm{Kg}_{20} & -\mathrm{B}_{\mathrm{d}} \mathrm{Kg}_{30} \\ \mathrm{~g}_{10} & \mathrm{~g}_{20} & \mathrm{~g}_{30} \\ \mathrm{~g}_{40} & \mathrm{~g}_{50} & \mathrm{~g}_{60} & \\ & & \end{array}\right] \mathrm{S}_{1}\right]>0$

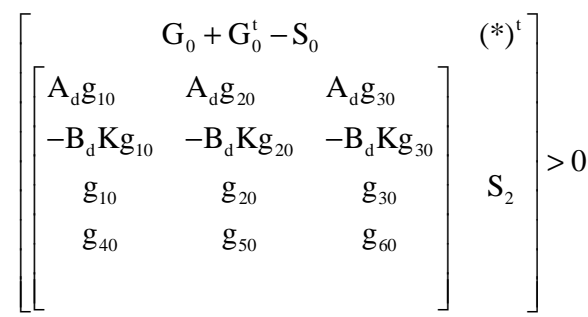

We verify Eq. 70:

$$
\begin{aligned}
\mathrm{G}_{2} & =\left[\begin{array}{lll}
\mathrm{g}_{12} & \mathrm{~g}_{22} & \mathrm{~g}_{32} \\
\mathrm{~g}_{42} & \mathrm{~g}_{52} & \mathrm{~g}_{62} \\
\mathrm{~g}_{72} & \mathrm{~g}_{82} & \mathrm{~g}_{92}
\end{array}\right] ; \\
\mathrm{G}_{1} & =\left[\begin{array}{lll}
\mathrm{g}_{11} & \mathrm{~g}_{21} & \mathrm{~g}_{31} \\
\mathrm{~g}_{41} & \mathrm{~g}_{51} & \mathrm{~g}_{61} \\
\mathrm{~g}_{71} & \mathrm{~g}_{81} & \mathrm{~g}_{91}
\end{array}\right] ; \mathrm{G}_{0}=\left[\begin{array}{lll}
\mathrm{g}_{10} & \mathrm{~g}_{20} & \mathrm{~g}_{30} \\
\mathrm{~g}_{40} & \mathrm{~g}_{50} & \mathrm{~g}_{60} \\
\mathrm{~g}_{70} & \mathrm{~g}_{80} & \mathrm{~g}_{90}
\end{array}\right]
\end{aligned}
$$

The previous conditions inequalities are BMI, difficult to resolve. We propose the following conditions:

$\mathrm{R}_{10}=\mathrm{Kg}_{10}, \mathrm{R}_{20}=\mathrm{Kg}_{20}, \mathrm{R}_{30}=\mathrm{Kg}_{30}, \mathrm{R}_{41}=\mathrm{Kg}_{41}$,

$\mathrm{R}_{51}=\mathrm{Kg}_{51}, \mathrm{R}_{61}=\mathrm{Kg}_{6}, \mathrm{R}_{32}=\mathrm{Kg}_{32}, \mathrm{R}_{72}=\mathrm{Kg}_{72}$,

$\mathrm{R}_{82}=\mathrm{Kg}_{82}$

$\mathrm{R}_{10}=\mathrm{R}_{20}=\mathrm{R}_{41}=\mathrm{R}_{61}=\mathrm{R}_{72}=\mathrm{R}_{82}=0$

$\mathrm{K}_{0}=\mathrm{R}_{30} \mathrm{~g}_{30}^{-1} \mathrm{~K}_{1}=\mathrm{R}_{51} \mathrm{~g}_{51}^{-1} \mathrm{~K}_{2}=\mathrm{R}_{92} \mathrm{~g}_{92}^{-1}$

\section{RESULTS AND DISCUSSION}

(66)

Illustration: The study of the stability of model states of the induction machine with delays in the order, based on the use of the second Lyapunov method and the formulation of the problem of calculating the gain matrix inequality leads to not linear. Thus the approach of system for the model of states of the induction machine is used to calculate the feedback gain $\mathrm{K}$ for a delay that happens has five times the sampling period $\mathrm{T}_{\mathrm{e}} . \mathrm{T}_{\mathrm{c}}=1 / \mathrm{f}_{\mathrm{c}}=100 \mu \mathrm{s} .\left(\mathrm{F}_{\mathrm{c}}=10 \mathrm{KHz}\right)$ To respect the Shannon theorem was, $\mathrm{T}_{\mathrm{e}}<2^{*} \mathrm{~T}_{\mathrm{c}}$ so we set $\mathrm{T}_{\mathrm{e}}=100 \mu \mathrm{s}$. The system in discrete time is that Eq. 71:

$\left\{\begin{array}{c}x(k+1)=A_{d} x(k)+B_{d} u(k) \\ y(k)=C_{d} x(k)+D_{d} u(k)\end{array}\right.$

With:

$$
\begin{aligned}
& \mathrm{A}_{\mathrm{d}}= \\
& {\left[\begin{array}{ccccc}
0.9812 & 0.03091 & 0.0113 & 2.726 & 0.001175 \\
-0.03095 & 0.981 & -0.728 & 0.01308 & 0.0108 \\
8.042 \mathrm{e}-05 & 1.512 \mathrm{e}-06 & 0.9994 & 0.001244 & -1.302 \mathrm{e}-05 \\
-1.123 \mathrm{e}-06 & 8.228 \mathrm{e}-05 & -000123 & 0.9994 & -0.0001195 \\
-0.002919 & -0.03137 & 0.3725 & 0.2837 & 0.9998
\end{array}\right]} \\
& \mathrm{B}_{\mathrm{d}}=\left[\begin{array}{ccc}
1.842 & -0.9853 & -0.9453 \\
-0.02891 & 1.608 & -1.579 \\
7.5 \mathrm{e}-05 & -3.666 \mathrm{e}-05 & -3.829 \mathrm{e}-05 \\
-6.496 \mathrm{e}-07 & 6.625 \mathrm{e}-05 & -6.55 \mathrm{e}-05 \\
-0.00287 & -0.02387 & 0.02674
\end{array}\right] \\
& \mathrm{C}_{\mathrm{d}}=\mathrm{C}, \mathrm{D}_{\mathrm{d}}=\mathrm{D}=0
\end{aligned}
$$


By applying the method of switching system to find the control matrix $\mathrm{K}$ such that the closed loop system for a delay is stable although determining and using Matlab for solving LMI inequality, we find the following results:

For a delay $\tau=1$ :

$$
\begin{aligned}
& \mathrm{K}_{1}=\mathrm{R}_{4} \mathrm{~g}_{4}^{-1}=1 \mathrm{e}-05^{*} \\
& {\left[\begin{array}{lllll}
0.1913 & 0.6358 & 0.0704 & -0.3410 & 0.3105 \\
0.1822 & 0.6079 & 0.0660 & -0.3280 & 0.2963 \\
0.1824 & 0.6067 & 0.0668 & -0.3259 & 0.2961
\end{array}\right]}
\end{aligned}
$$

For a delay $\tau=2$ :

$$
\begin{aligned}
& \mathrm{K}_{2}=\mathrm{R}_{9} \mathrm{~g}_{9}^{-1}=1 \mathrm{e}-04 * \\
& {\left[\begin{array}{lllll}
0.0716 & 0.1180 & 0.1069 & 0.0077 & 0.0521 \\
0.0684 & 0.1127 & 0.1020 & 0.0073 & 0.0497 \\
0.0683 & 0.1125 & 0.1020 & 0.0073 & 0.0497
\end{array}\right]}
\end{aligned}
$$

For a delay $\tau=3$ :

$$
\begin{aligned}
& \mathrm{K}_{2}=\mathrm{R}_{16} \mathrm{~g}_{16}^{-1}=1 \mathrm{e}-05^{*} \\
& {\left[\begin{array}{lllll}
-0.1840 & -0.1790 & 0.3989 & 0.0615 & -0.2385 \\
-0.1755 & -0.1708 & 0.3807 & 0.0586 & -0.2277 \\
-0.1755 & -0.1707 & 0.3805 & 0.0587 & -0.2275
\end{array}\right]}
\end{aligned}
$$

\section{For a delay $\tau=4$ :}

$$
\begin{aligned}
& \mathrm{K}_{4}=\mathrm{R}_{25} \mathrm{~g}_{25}^{-1}=1 \mathrm{e}-04 * \\
& {\left[\begin{array}{ccccc}
-0.1101 & -0.1022 & -0.1772 & -0.1599 & -0.942 \\
-0.1052 & -0.0976 & -0.1692 & -0.1527 & -0.0899 \\
-0.1051 & -0.975 & -0.1690 & -0.1526 & -0.0898
\end{array}\right]}
\end{aligned}
$$

\section{For a delay $\tau=5$ :}

$$
\begin{aligned}
& \mathrm{K}_{5}=\mathrm{R}_{36} \mathrm{~g}_{36}^{-1}=1.0 \mathrm{e}-08 * \\
& {\left[\begin{array}{lllll}
0.0475 & 0.1102 & 0.0358 & -0.0133 & 0.0564 \\
0.0453 & 0.1052 & 0.0342 & -0.0127 & 0.0538 \\
0.0453 & 0.1051 & 0.0342 & -0.0127 & 0.0537
\end{array}\right]}
\end{aligned}
$$

\section{For a delay: $\tau \in[0,2]$ :}

$$
\begin{aligned}
& \mathrm{K}_{0}=\mathrm{R}_{30} \mathrm{~g}_{30}^{-1}=1.0 \mathrm{e}-004 * \\
& {\left[\begin{array}{lllll}
-0.1365 & 0.0456 & 0.1375 & 0.6665 & 1.7229 \\
-0.1303 & 0.0436 & 0.1313 & 0.6364 & 1.6450 \\
-0.1302 & 0.0435 & 0.1312 & 0.6358 & 1.6435
\end{array}\right]}
\end{aligned}
$$

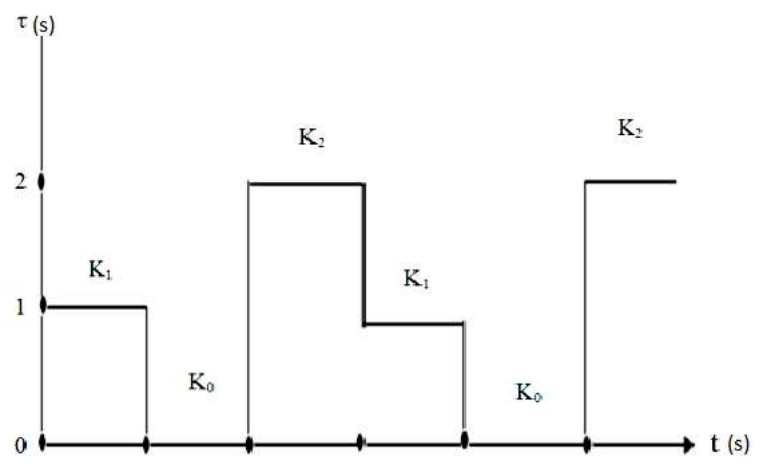

Fig. 1: Switching gain $\mathrm{K}$ according to the delay
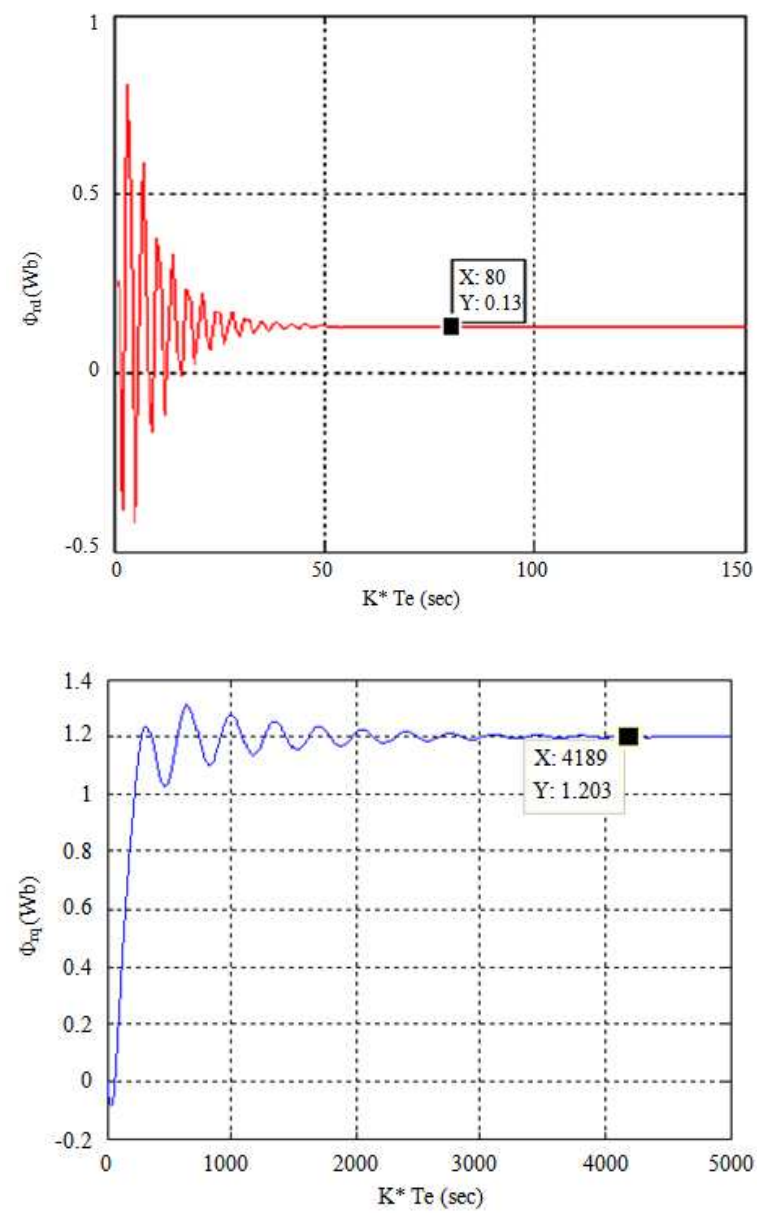

Fig. 2: Nominal Rotor flux along the axis "d" and axis "q"

$$
\begin{aligned}
& \mathrm{K}_{1}=\mathrm{R}_{51} \mathrm{~g}_{51}^{-1}=1.0 \mathrm{e}-003 * \\
& {\left[\begin{array}{lllll}
-0.3695 & 1.1142 & 0.2694 & 0.0574 & -1.2551 \\
-0.3528 & 1.0640 & 0.2573 & 0.0574 & -1.1985 \\
-0.3525 & 1.0629 & 0.2570 & 0.0548 & -1.1973
\end{array}\right]}
\end{aligned}
$$




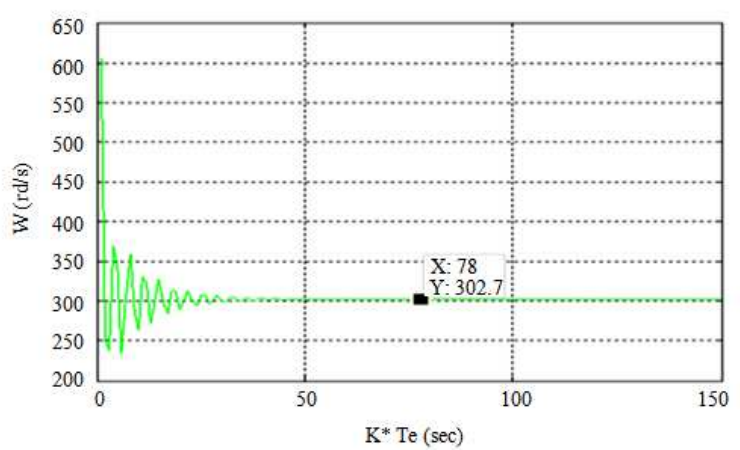

Fig. 3: Rated electric pulsation

$$
\begin{aligned}
& \mathrm{K}_{2}=\mathrm{R}_{92} \mathrm{~g}_{92}^{-1}= \\
& {\left[\begin{array}{lllll}
0.3945 & 0.4119 & 0.4171 & 0.3819 & 0.4143 \\
0.3766 & 0.3933 & 0.3983 & 0.3646 & 0.3955 \\
0.3763 & 0.3929 & 0.3979 & 0.3643 & 0.3952
\end{array}\right]}
\end{aligned}
$$

Simulation and results: Matlab simulation of the system around the nominal operating point below for a delay $\tau \in[0,2]$, (Fig. 1) gives the following signals:

The nominal operating point: $\left\{\begin{array}{l}\operatorname{Isd}_{\mathrm{n}}=10.97 \mathrm{~A} \\ \mathrm{Isq}_{\mathrm{n}}=15.77 \mathrm{~A} \\ \varnothing \mathrm{rd}_{\mathrm{n}}=1.2 \mathrm{~Wb} \\ \varnothing \mathrm{rq}_{\mathrm{n}}=0.13 \mathrm{~Wb} \\ \mathrm{~W}_{\mathrm{n}}=302.6 \mathrm{rd} / \mathrm{s}\end{array}\right.$

According to the curves of the outputs $\Phi_{\mathrm{rq}}, \Phi_{\mathrm{rd}}$ and $\omega$ system we find that the system is stable for a delay $\tau$ $=2$, (Fig. 2 and 3), the state feedback gain $\mathrm{K}_{2}$ calculated by the method of switching system stabilizes the closed loop system for a delay of less than two sampling periods. This calculation method therefore offers a large range of stability because it tolerates delays in transmission networks $0 \leq \tau \leq 2$.

\section{CONCLUSION}

Remote control with stability problems is a new challenge for scientists. Indeed, despite the progress the case of nonlinear systems with remote-controlled continuous delays pose serious problems of stability and stabilization.

In this study, we proposed stabilization algorithms based on the use of hopping systems for the calculation of a gain stabilizing state feedback. The difficulty of this approach is to obtain non-linear matrix. Inequalities require the contribution of relaxation that can reduce the field of possible solutions. The proposed method was tested on an induction machine supplied by a variable speed drive, despite the random delays introduced the system remains stable and retains the nominal performance.

\section{REFERENCES}

Apkarian, P. and H.D. Tuan, 2000. Robust control via concave minimization local and global algorithms. IEEE Trans. Automatic Control, 45: 299-305. DOI: $10.1109 / 9.839953$

Ariba, Y. and F. Gouaisbaut, 2007. Delay-dependent stability analysis of linear systems with timevarying delay. Proceedings of the 46th IEEE Conference on Decision and Control, Dec. 12-14, IEEE Xplore Press, New Orleans, LA., pp: 20532058. DOI: 10.1109/CDC.2007.4434619

Benchohra, M., J. Henderson, S.K. Ntouyas, A. Ouahab, 2007. Existence results for fractional order functional differential equations with infinite delay. J. Math. Anal. Appli., 338: 1340-1350. DOI: 10.1016/j.jmaa.2007.06.021

Boucetta, A., 2008. Vector control of a variable reluctance machine stator and rotor discs imbricates. Am. J. Eng. Applied Sci., 1: 260-265. DOI: 10.3844/ajeassp.2008.260.265

Castillo-Toledoy, B., S.D. Gennaroz, A.G. Loukianovy and J. Riveray, 2004. On the discrete-time modelling and control of induction motors with sliding modes. Proceedings of the 2004 American Control Conference, Jun. 30-Jul. 2, IEEE Xplore Press, Boston, MA, USA., pp: 2598-2602.

Cloosterman, M.B.G., N.V.D. Wouw, W.P.M. Heemels and H. Nijmeijer, 2007. Stability of networked control systems with large delays. Proceedings of the 46th IEEE Conference on Decision and Control, Dec. 12-14, IEEE Xplore Press, New Orleans, LA., pp: 5017-5022. DOI: 10.1109/CDC.2007.4434669

Daafouz, J. and J. Bernussou, 2001. Parameter dependent lyapunov functions for discrete time systems with time varying parametric uncertainties. Syst. Control Lett., 43: 355-359. DOI: 10.1016/S0167-6911(01)00118-9

Fridman, E. and U. Shaked, 2002. An improved stabilization method for linear time-delay systems, IEEE Trans. Automatic Control, 47: 1931-1937. DOI: $10.1109 /$ TAC.2002.804462 
Gouaisbaut, F. and D. Peaucelle, 2006. A note on Stability of Time Delay systems. Proceedings of the 5th IFAC Symposium on Robust Control Design, Jul. 5-7, Toulouse, France, pp: 1-6.

Gouaisbaut, F., 2005. Stability and stabilization of distributed time delay systems. Proceedings of the 44th IEEE Conference on Decision and Control and European Control Conference, Dec. 12-15, IEEE Xplore Press, pp: 1379-1384. DOI: 10.1109/CDC.2005.1582351

Gouaisbaut, F.and Y. Ariba, 2009. Delay range stability of distributed time delay systems. Proceedings of the 6th IFAC Symposium on Robust Control Design, (SRCD' 09), IFAC, Israel. DOI: 10.3182/20090616-3-IL-2002.00039

Henrion, D., S. Tarbouriech and M. Sebek, 1999. Rankone LMI approach to simultaneous stabilization of linear systems. Syst. Control Lett., 38: 79-89. DOI: 10.1016/S0167-6911(99)00049-3

Hetel, L., J. Daafouz and C. Lung, 2007. Stability analysis for discrete time switched systems with temporary uncertain switching signal. Proceedings of the 46th IEEE Conference on Decision and Control, Dec. 12-14, IEEE Xplore Press, New Orleans, LA., pp: 5623-5628. DOI: 10.1109/CDC.2007.4434794
Hu, L., Y. Sun and Y. Cao, 1998. Robust control for uncertain linear systems with constraints on output. Proceedings of the American Control Conference, Jun. 21-26, IEEE Xplore Press, Philadelphia, PA, USA., pp: 2708-2712. DOI: 10.1109/ACC.1998.688342

Kechiche, O.B.H.B., H.B.A. Sethom, H. Sammoud and I.S. Belkhodja, 2011. Optimized high frequency signal injection based permanent magnet synchronous motor rotor position estimation applied to washing machines. Am. J. Eng. Applied Sci., 4 4: 390-399. DOI: 10.3844/ajeassp.2011.390.399

Labit, Y., Y. Ariba and F. Gouaisbaut, 2007. Design of Lyapunov based controllers as TCP AQM. LAASCNRS, University of Toulouse, France.

Migloire, E.G., 2004. Predictive control based on semidefinite programming. National Institute of Applied Sciences, Toulouse.

Teixeira, M.C.M., E. Assuncao and R.G. Avellar, 2003. On relaxed LMI-based designs for fuzzy regulators and fuzzy observers. IEEE Trans. Fuzzy Syst., 11: 613-623. DOI: 10.1109/TFUZZ.2003.817840

Zhu, Q. and G.D. Hu, 2009. Stability analysis for uncertain nonlinear time-delay systems with quasione-sided lipschitz condition. Acta Automatica Sinica, 35: 1006-1009. DOI: 10.1016/S18741029(08)60099-3 